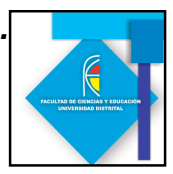

\title{
MOVILIDAD DE LAS REPRESENTACIONES DEL MODELO SOL - TIERRA - LUNA: ESTRATEGIA DIDÁCTICA PARA ESTUDIANTES DE QUINTO DE PRIMARIA.
}

\section{MOBILITY MODEL REPRESENTATIONS OF SUN - EARTH - MOON: STUDENT TEAC HING STRATEGY FIFTH GRADE.}

\section{Resumen}

\author{
Maritza Menjura Gonzalez ${ }^{1}$ \\ Mónica Jhoanna Ochoa Cárdenas ${ }^{2}$ \\ Rusby Malangón ${ }^{3}$ \\ Nidia Tuay ${ }^{4}$
}

En el ámbito de la enseñanza de las ciencias, la astronomía ha sido un tema de gran interés ya que ha servido como puente entre el gusto individual del hombre por los fenómenos naturales y el conocimiento científico. Por esta razón el presente trabajo busca aprovechar la curiosidad que despierta en los niños los fenómenos físicos implícitos en los cuerpos celestes que diariamente observan, para llevarlos al estudio del modelo heliocéntrico. Para lograr el objetivo propuesto, inicialmente se identifican las representaciones que estructuran los estudiantes de quinto de primaria del Colegio José Martí IED, en relación al sistema Sol-Tierra-Luna, con el fin de diseñar una estrategia didáctica que posibilite la movilidad de estas representaciones, orientarlas o fortalecerlas para la comprensión del modelo heliocéntrico, a partir del estudio de las fases de la luna. Este trabajo investigativo parte por reconocer, a los estudiantes de primaria como pequeños exploradores dotados de las habilidades para explicar con argumentos el mundo que los rodea. La estrategia diseñada favorece la construcción de nociones científicas que posteriormente se convertirán en un eslabón que favorecerá el aprendizaje de las ciencias durante el proceso académico.

Palabras clave: enseñanza, aprendizaje, representaciones, actividades didácticas.

\section{Abstract}

In the field of science education, astronomy has been a topic of great interest, which has served as a bridge between the individual man's taste by natural phenomena and scientific knowledge. Therefore this paper seeks to take advantage of the curiosity

\footnotetext{
${ }^{1}$ Estudiante de licenciatura en física Universidad Pedagógica Nacional, dfi536_mmenjura@pedagogica.edu.co

${ }^{2}$ Estudiante de licenciatura en física Universidad Pedagógica Nacional

${ }^{3}$ Estudiante de licenciatura en física Universidad Pedagógica Nacional

${ }^{4}$ Docente Universidad Pedagógica Nacional.
} 


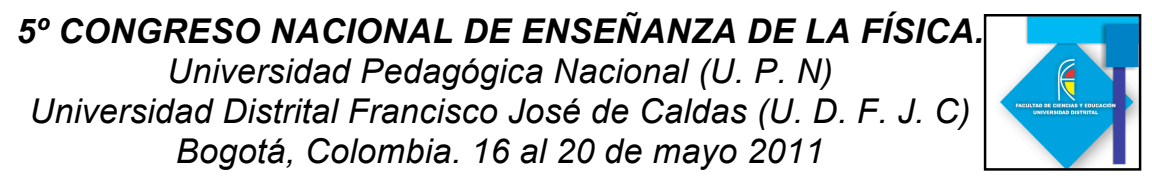

aroused in children by the celestial bodies observed daily to put forward the study of the heliocentric model. To achieve this objective, first we identified the representations about the Sun-earth-moon system of the fifth-grade students of the College José Martí IED, in order to design a teaching strategy that facilitates the mobility of these representations, to guide them or to strength them for the understanding of the heliocentric model, based on a study of the phases of the moon. This research paper begins by recognizing the students of elementary school as young explorers equipped with the skills to explain with arguments the world around them. This strategy pretends to favor the construction of scientific concepts which later will become a link that will promote the learning of science in academic processes.

Keywords: teaching, learning, representations, didactic activities.

\section{Introducción}

Al realizar una observación sistemática en el colegio José Martí IED, en los cursos de básica primaria, como cuarto y quinto, se logro identificar una actitud activa en los niños frente al aprendizaje de los fenómenos naturales. Los estudiantes en esta edad son exploradores por naturaleza, manifestando curiosidad e interés por conocer las respuestas de los fenómenos que observan, haciendo preguntas como ¿Por qué el cielo es azul?, ¿Por qué la luna no se cae?, si la tierra es redonda, ¿Por qué el agua de los océanos no se derrama? entre otras.

Desafortunadamente en la escuela, las preguntas que hacen los niños sobre los fenómenos naturales no son de gran relevancia, ya que en algunos casos como el Colegio José Martí, no cuenta con profesores de la básica primaria, especializados en el área de ciencias, que puedan orientar las preguntas de los niños que por lo general están relacionadas con la Astronomía. Esto se debe a que las maestras de la básica primaria deben dividir su jornada escolar para explicar las diferentes áreas de conocimiento que el Ministerio de Educación Nacional establece como fundamentales para la enseñanza en primaria. Por lo tanto, los temas en ciencias son vistos superficialmente sin importar la pertinencia del tema con lo que el estudiante necesita aprender, para darle respuesta a sus preguntas y orientar las representaciones que el niño por naturaleza estructura de su entorno en este caso del sistema Sol-Tierra-Luna.

Considerando la importancia de las representaciones de los niños, es necesario ahondar en los modelos que tienen ellos, con el fin de valorarlos e identificar las representaciones que poseen sobre los fenómenos celestes, y construir una propuesta didáctica que permita la movilidad de estas representaciones hacia un modelo heliocéntrico. De esta manera, toma importancia las preguntas: ¿Cómo generar actitudes favorables hacia el estudio de la ciencia desde los primeros años escolares? Y ¿Cómo aproximar a los estudiantes del colegio José Martí IED del grado cuarto de primaria a la comprensión del modelo heliocéntrico a partir del estudio de las fases de la luna?

Se propone una alternativa de trabajo didáctico para promover actitudes favorables en los estudiantes de la básica primaria. Ya que se evidencia que la escuela da mayor importancia y pertinencia al abordaje de las ciencias naturales en la básica secundaria y media que en la básica primaria, subestimando los procesos cognitivos que realizan los 


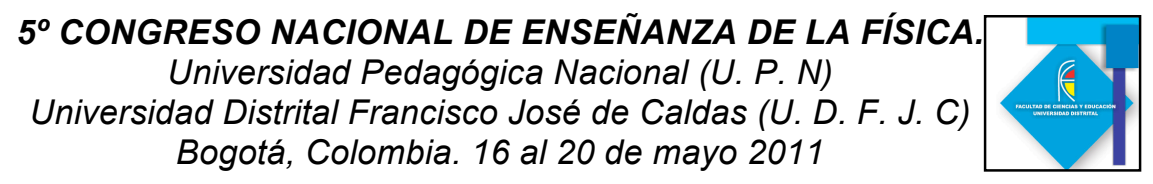

niños respecto a los fenómenos naturales y olvidando que los estándares propuestos por el MEN consideran al niño como un científico natural. El conocimiento científico debe comenzar a construirse desde la infancia pues esto permite fomentar la curiosidad, la sensibilidad y capacidad de asombro, además el paulatino desarrollo de las habilidades del pensamiento respecto a los fenómenos físicos, para que posteriormente en la secundaria el estudiante no sea atropellado con modelos matemáticos y conceptos que requieren de un alto nivel de "imaginación" y abstracción generándose una actitud de indiferencia por parte de los estudiantes, hacia el aprendizaje de las ciencias. Tal como lo expresan (Campos, Galván, Campos \& Rodríguez, sin fecha de publicación):

"La dedicación a los temas científicos en la escuela primaria, ha tenido un papel menos importante que a nivel secundaria, sin embargo, es trascendental que los profesores seamos conscientes de la necesidad de las experiencias específicamente cientificas que puedan favorecer el desarrollo integral de los alumnos. Se observa que los niños pequeños lo preguntan todo $y$, a medida que transitan por los diferentes grados de la escuela, cada vez preguntan menos e, incluso, llegan a no preguntar en absoluto".

Se considera importante realizar un trabajo investigativo centrado en la didáctica de las ciencias para la escuela primaria, a partir del estudio de la astronomía. Por cuanto, es un tema que estimula la curiosidad e interés de los niños por la ciencia, induciendo el razonamiento, la investigación y la creatividad en el aula, fomentando en los estudiantes emociones y sensaciones que los motiva a querer saber más, con enfoques al desarrollo del pensamiento científico. (Polanco, 2009) menciona en su artículo que la astronomía ha sido un tema de interés para el hombre a lo largo de la historia, donde este ha desarrollado el conocimiento y la comprensión de los movimientos de cuerpos celestes, construyendo por medio de investigaciones y observaciones modelos astronómicos que les permitía dar una representación científica a las preocupaciones de su época.

Tomando en consideración la evolución de los modelos astronómicos, es importante reflexionar como hoy en día los niños construyen sus representaciones sobre el sistema sol-tierra-luna, teniendo en cuenta sus características perceptuales del mundo, tal como lo menciona (Pedrinaci, 2009)

"es verdad que nuestros sentidos nos dicen cada día que la tierra esta quieta mientras que el sol gira alrededor de nosotros, y que esta falsa evidencia de sentido común, como muestra la historia de la ciencia, ha hecho muy difícil superar el sistema geocéntrico".

Por esta razón se encontró pertinente abordar el tema de astronomía que se centra en la compresión del modelo heliocéntrico a partir del estudio de las fases de la luna, para ayudar a los niños a desarrollar gradualmente la comprensión de modelos mentales acordes con la ciencia.

\section{Propuesta didáctica}

Para lograr el objetivo presentado, se procede a diseñar la propuesta didáctica que posibilitará la movilidad de las representaciones intuitivas a las representaciones científicas en los estudiantes, sobre el modelo heliocéntrico a partir del estudio de las fases de la Luna. Inicialmente se realizó un diagnostico de la población que permitió reconocerla en 


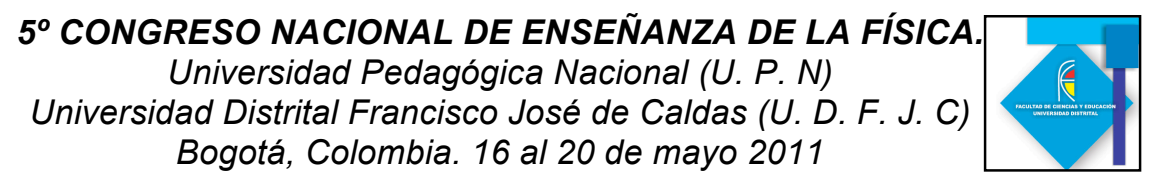

términos socio-culturales y cognitivos. Se identifico que la mayoría de los estudiantes del Colegio José Martí IED ubicado en la localidad 18 con estratos 1 y 2 , presentan problemas en su convivencia, dificultades en el proceso de aprendizaje, su expresión verbal y física con sus compañeros es violenta, a demás de presentar actitud de rebeldía hacia sus docentes. Pero también se identificó que aunque hay niños agresivos en el aula, tal vez por la influencia de su entorno social, igualmente hay niños muy respetuosos que aunque se encuentran rodeados de factores que amenazan su salud física y mental, son alegres y con muchas ganas de aprender. Una vez realizado este diagnóstico se dió inicio a la construcción de la estrategia didáctica la cual se sustenta en 5 ejes centrales, mostrados en la tabla 1.

\begin{tabular}{|c|l|}
\hline $\begin{array}{c}\text { Habilidades de pensamiento } \\
\text { científico }\end{array}$ & Observar, identificar, comparar, analizar. \\
\hline $\begin{array}{c}\text { El uso de representaciones y } \\
\text { modelos en la enseñanza de las } \\
\text { ciencias }\end{array}$ & $\begin{array}{l}\text { Los estudiantes construyen modelos mentales para representar lo que } \\
\text { perciben del mundo y estos constituyen la base sobre la cual se } \\
\text { emprenden las acciones pedagógicas que permitirán la comprensión } \\
\text { de los modelos conceptuales elaborados por la ciencia. }\end{array}$ \\
\hline $\begin{array}{c}\text { Importancia de los conocimientos } \\
\text { previos según Ausubel en la teoría } \\
\text { del aprendizaje significativo. }\end{array}$ & $\begin{array}{l}\text { Para Ausubel, los conocimientos previos de los estudiantes deben ser } \\
\text { el punto de partida para la iniciación de un proceso de enseñanza- } \\
\text { aprendizaje en el ámbito escolar y no se puede despreciar el } \\
\text { conocimiento científico alternativo que el individuo ha logrado } \\
\text { desarrollar }\end{array}$ \\
\hline $\begin{array}{c}\text { Importancia del lenguaje según } \\
\text { Vygotsky }\end{array}$ & $\begin{array}{l}\text { El lenguaje cobra un papel importante en el desarrollo cognitivo, ya } \\
\text { que este es la principal vía de transmisión de la cultura y expresa el } \\
\text { pensamiento. }\end{array}$ \\
\hline El papel de la pregunta en el \\
aprendizaje & $\begin{array}{l}\text { La pregunta es una herramienta que permite exponer ideas, indagar, } \\
\text { confrontar y desarrollar procesos de formación autónoma y critica } \\
\text { durante el aprendizaje. }\end{array}$ \\
\hline
\end{tabular}

Tabla 1: ejes centrales de la propuesta

A continuación se expone de manera resumida la ruta sugerida, la cual contiene 3 sesiones que se subdividen en diferentes momentos de aprendizaje, cada momento está compuesto por una o varias actividades con diferentes propósitos.

La sesión 1 denominada "explorando saberes", está conformada por dos momentos, cada momento contiene una actividad, mediante las cuales se busca identificar las representaciones intuitivas que poseen los estudiantes sobre el modelo astronómico que se pretende abordar.

La segunda sesión se nombra "Estructurando saberes", está conformada por dos momentos. El momento 1 contiene 3 actividades a partir de las cuales se pretende estimular las habilidades de pensamiento, observar, identificar, comparar y analizar. En el segundo momento se presentan tres actividades que buscan recrear situaciones con el fin de llevar a los estudiantes a la comprensión del modelo heliocéntrico.

La tercera y última sesión denominada "evaluando saberes", contiene solo un momento, en el cual se proponen dos actividades que buscan generar un conflicto cognitivo en el estudiante, con el fin de que el haga uso de sus nuevos conocimientos a través de un modelo explicativo conceptual. En las sesiones 2 y 3 se trabaja constantemente la habilidad de la observación, ya que esta capacidad es la que encabeza el proceso de investigación científica. La organización de la estrategia didáctica se muestra en la tabla 2. 
$5^{\circ}$ CONGRESO NACIONAL DE ENSEÑANZA DE LA FÍSICA.

Universidad Pedagógica Nacional (U. P. N)

Universidad Distrital Francisco José de Caldas (U. D. F. J. C)

Bogotá, Colombia. 16 al 20 de mayo 2011

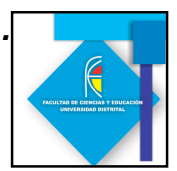

\begin{tabular}{|c|c|c|}
\hline \multirow[t]{2}{*}{ Sesión 1: Explorando saberes } & Momentos & Actividades \\
\hline & \multirow{2}{*}{$\begin{array}{l}\text { Momento 1: Reconociendo tus ideas } \\
\text { Propósito: Identificar el modelo explicativo que el } \\
\text { estudiante utiliza para argumentar su } \\
\text { representación }\end{array}$} & \multirow{2}{*}{$\begin{array}{l}\text { ¿Cómo te } \\
\text { imaginas el } \\
\text { universo? }\end{array}$} \\
\hline \multirow[t]{2}{*}{$\begin{array}{l}\text { Propósito: } \quad \text { Identificar } \quad \text { las } \\
\text { representaciones que tienen los niños } \\
\text { del sistema Sol-Tierra-Luna. }\end{array}$} & & \\
\hline & $\begin{array}{l}\text { Momento 2: Explorando la imaginación } \\
\text { Propósito: Llevar a los estudiantes a Identificar las } \\
\text { explicaciones que construyen dos personajes de un } \\
\text { cuento frente al comportamiento del sistema Sol } \\
\text { Tierra Luna y a asumir una postura de acuerdo o } \\
\text { desacuerdo frente a estas explicaciones. Esto, con } \\
\text { el propósito de identificar los modelos explicativos } \\
\text { de los estudiantes. }\end{array}$ & $\begin{array}{l}\text { "El universo es } \\
\text { un cuento:" }\end{array}$ \\
\hline \multirow{3}{*}{$\begin{array}{l}\text { Sesión 2: Estructurando saberes } \\
\text { Propósito: Desarrollar en los niños la } \\
\text { habilidad de observar de manera } \\
\text { sistemática, el movimiento y apariencia } \\
\text { de la luna, para así, motivarlos a } \\
\text { establecer un modelo explicativo } \\
\text { acerca de la relación que existe entre } \\
\text { los tres cuerpos celestes: Luna, Tierra } \\
\text { y Sol, basado en las observaciones } \\
\text { realizadas y en el análisis de las } \\
\text { mismas. }\end{array}$} & \multirow{3}{*}{$\begin{array}{l}\text { Momento 1: Descubriendo y fomentando el } \\
\text { espíritu científico } \\
\text { Propósito 1: Estimular en los estudiantes las } \\
\text { habilidades de pensamiento como: Observar, } \\
\text { identificar, y comparar promoviendo la } \\
\text { investigación científica en ellos. } \\
\text { Propósito 2: Motivar a los estudiantes a que } \\
\text { retomen la observación sistemática que han hecho } \\
\text { de la Luna y a partir de esta, reflexionar sobre las } \\
\text { diferencias y similitudes que han encontrado } \\
\text { diariamente, para que logren identificar que siempre } \\
\text { vemos la misma cara de la Luna y comprender por } \\
\text { qué sucede esto. }\end{array}$} & \multirow[b]{2}{*}{$\begin{array}{l}\text { Espiando a la } \\
\text { Luna }\end{array}$} \\
\hline & & \\
\hline & & $\begin{array}{l}\text { El secreto de } \\
\text { la Luna }\end{array}$ \\
\hline & $\begin{array}{l}\text { Momento 2: descubriendo y aprendiendo } \\
\text { Propósito: Disponer situaciones didácticas: } \\
\text { observar fenómenos, recrear situaciones, formular }\end{array}$ & $\begin{array}{c}\text { ¿Por qué } \\
\text { cambia la } \\
\text { Luna? }\end{array}$ \\
\hline & $\begin{array}{l}\text { preguntas etc. para llevar a los estudiantes a la } \\
\text { comprensión del modelo heliocéntrico. }\end{array}$ & $\begin{array}{l}\text { La Tierra, el } \\
\text { Sol y la Luna }\end{array}$ \\
\hline & & La elipse \\
\hline Sesión 3: evaluando saberes & Momento 1: Que aprendimos & Rastreando al \\
\hline $\begin{array}{l}\text { Propósito: Retomar las explicaciones } \\
\text { dadas por los estudiantes en la sesión }\end{array}$ & $\begin{array}{l}\text { Propósito: Estimular en los estudiantes la habilidad } \\
\text { de la observación a partir de seguirle el rastro al sol }\end{array}$ & \\
\hline $\begin{array}{l}\text { 1, para contrastarlas con los nuevos } \\
\text { saberes y así comprobar si se logró } \\
\text { llevar a los estudiantes a una movilidad } \\
\text { de representaciones. Su } \\
\text { argumentación y el uso del lenguaje de } \\
\text { la ciencia serán algunos de los criterios } \\
\text { de seguimiento. }\end{array}$ & $\begin{array}{l}\text { en diferentes momentos del dla y llevario a } \\
\text { construir modelos explicativos desde las } \\
\text { observaciones realizadas. }\end{array}$ & $\begin{array}{l}\text { Actividad de } \\
\text { cierre }\end{array}$ \\
\hline
\end{tabular}

Tabla 2: Bosquejo de la Estrategia Didáctica

\section{Conclusiones}

Hasta el momento el mayor logro alcanzado con la propuesta didáctica, es haber conseguido que los niños revivan la historia de la astronomía, repitiendo las técnicas utilizadas por los pensadores de la antigüedad, como es el método de la observación sistemática. Se ha reflexionado con ellos sobre la importancia de la observación y cómo ésta ha contribuido significativamente al desarrollo del conocimiento científico. Por otro lado, por medio de la ruta de aprendizaje propuesta. Se ha logrado despertar en los niños 


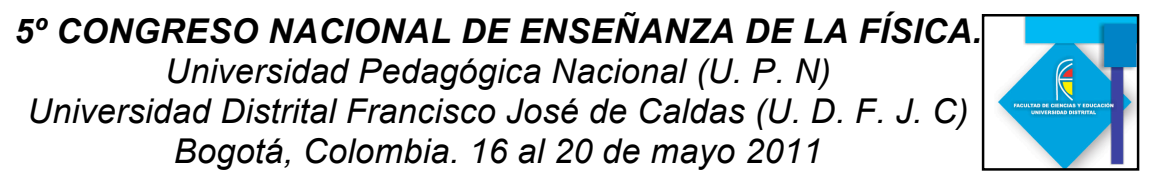

la curiosidad por conocer y entender el mundo físico que los rodea, como es el caso del fenómeno de las fases de la Luna, manteniendo viva la motivación e interés de la mayoría y la disposición subjetiva para el aprendizaje.

\section{Referencias Bibliográficas}

Pedrinaci, E. (2009). 2009, un año internacional de la ciencia, Alambique, 61, 5-8.

Polanco, F.J. (2009). El problema de la posición de la tierra en el universo. Sistemas geocéntrico y heliocéntrico, en Contribuciones a las Ciencias Sociales.

www.eumed.net/rev/cccss/06/fjpl3.htm 